\title{
CONTACT PROBLEMS FOR INTERFACE CRACKS UNDER HARMONIC SHEAR LOADING
}

\author{
VASYL A. MENSHYKOV ${ }^{1}$, OLEKSANDR V. MENSHYKOV² AND IGOR A. GUZ ${ }^{3}$ \\ ${ }^{1}$ National Aerospace University - "Kharkiv Aviation Institute" \\ Chkalova St, 17, Kharkiv 61000, Ukraine \\ v.menshykov@khai.edu.ua
}

${ }^{2}$ Centre for Micro- and Nanomechanics, School of Engineering, University of Aberdeen AB24 3UE Aberdeen, Scotland, UK

o.menshykov@abdn.ac.uk, https://www.abdn.ac.uk/engineering/people/profiles/o.menshykov

${ }^{3}$ Centre for Micro- and Nanomechanics, School of Engineering, University of Aberdeen AB24 3UE Aberdeen, Scotland, UK

i.guz@abdn.ac.uk, https://www.abdn.ac.uk/engineering/people/profiles/i.guz

Key words: Interface Crack Closure, Boundary Integrals, Friction.

Summary. The linear crack between two dissimilar elastic isotropic half-spaces under normal harmonic shear loading is considered. To take the crack faces interaction into account we assumed that the contact satisfies the Signorini constraints and the Coulomb friction law. The problem is solved numerically using the iterative process - the solution changes until the distribution of physical values satisfying the contact constraints is found. The numerical convergence of the method with respect to the number of the Fourier coefficients and mesh size is analysed. The effects of material properties and values of the friction coefficient on the distribution of displacements and contact forces are presented and analysed. Special attention is paid to the size of the contact zone and the results are compared with the classical model solutions obtained for the static problems with and without friction.

\section{INTRODUCTION}

Since all existing engineering materials contain cracks, notches, delaminations and other crack-like defects, a considerable body of work is devoted to the solution of two- and threedimensional fracture mechanics problems for cracked materials under static and dynamic loading [1-12].

It shall be specifically noted that the opposite faces of the existing cracks almost always interact with each other under deformation, significantly changing the solution near the crack [13-23]. The nature of the dynamic contact interaction between opposite crack faces is very complex. Under deformation of the material the initial contact region changes in time; its shape is unknown beforehand and must be determined as a part of the solution. The complexity of the problem is further compounded by the fact that the contact behaviour is very sensitive to the material properties of the two contacting surfaces and parameters of the external loading. It is 
only possible to solve these problems using advanced numerical methods, since the analytical solutions are limited to a relatively small number of idealized model problems corresponding to very special geometrical configurations and loading conditions. Consequently, due to the nonlinearity of the problem and substantial computational difficulties, researchers often neglect effects of crack faces contact interaction, for comprehensive reviews please see $[16,17]$.

The current study is devoted to the analysis of the size of the contact zone for the interface crack under shear loading. The results are obtained taking friction into account and completely disregarding it using the iterative algorithm [24], and the solution is compared with the classical model solutions for the static problems with and without friction [25].

\section{STATEMENT OF THE PROBLEM}

Let us consider an elastic bimaterial consisting of two homogeneous isotropic half-spaces, $\Omega^{(1)}$ and $\Omega^{(2)}$, with the crack of finite length located at the interface between half-spaces. The crack has no initial opening, and the normal harmonic shear loading with the frequency of $\omega=$ $2 \pi / T$ is applied.

In both half-spaces the equation of motion and the generalized Hooke's law lead to the linear Lamé equations of elastodynamics for the displacement field with the standard initial and boundary conditions for displacements and stresses (namely, no initial deformations; given initial load at the crack faces, $\Gamma^{(1) c r}$ and $\Gamma^{(2) c r}$; continuity conditions at the bonding interface,

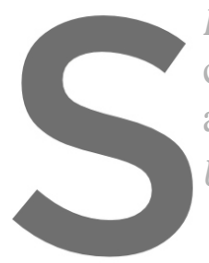
$\Gamma^{*}=\Gamma^{(1)} \cap \Gamma^{(2)}$; and the
components of the disp
and tractions using the $\mathrm{S}$
$U_{i j}^{(m)}(\mathbf{x}, \mathbf{y}, t-\tau)$ and $W_{i j}^{(}$
\[ u_{j}^{(m)}(\mathbf{x}, t)=\int_{\Gamma(m)} \]
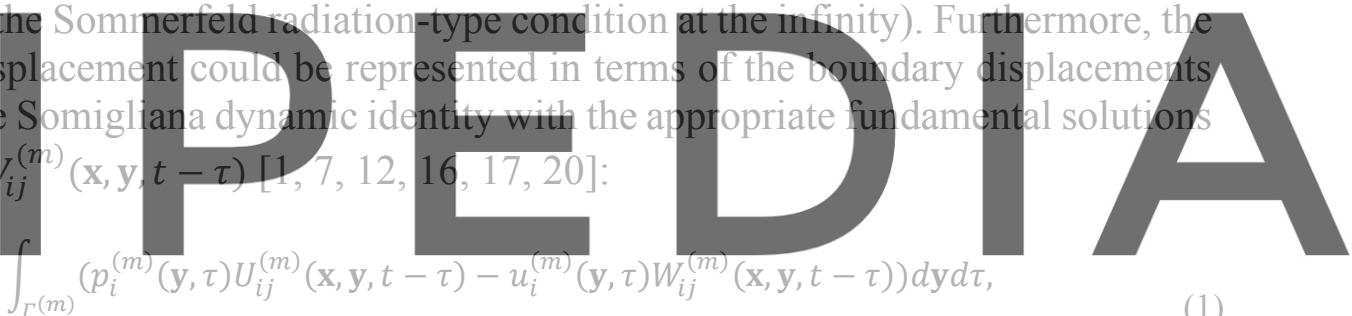

(1)

Register for free at https//www.scipedia.çam to down Load the version without the watermark

As it was mentioned before, due to the crack's closure the traction vector at the crack surface is the superposition of the initial traction caused by the incident load, $\mathbf{g}(\mathbf{x}, t)$, and the contact force, $\mathbf{q}(\mathbf{x}, t)$, that arises in the contact region, which is unknown beforehand, depends on the properties of the bimaterial and the friction coefficient and must be determined as a part of the solution.

To include the contact interaction into account, the Signorini unilateral constraints and the Coulomb friction law with the friction coefficient $k_{\tau}$ must be imposed for the normal and tangential components of the displacement discontinuity, $[\mathbf{u}(\mathbf{x}, t)]=\mathbf{u}^{(1)}(\mathbf{x}, t)-\mathbf{u}^{(2)}(\mathbf{x}, t)$, and contact forces:

$$
\begin{gathered}
{\left[u_{n}(\mathbf{x}, t)\right] \geq 0, \quad q_{n}(\mathbf{x}, t) \geq 0, \quad\left[u_{n}(\mathbf{x}, t)\right] q_{n}(\mathbf{x}, t)=0,} \\
\left|q_{\tau}(\mathbf{x}, t)\right|<k_{\tau} q_{n}(\mathbf{x}, t) \Rightarrow \frac{\partial\left[u_{\tau}(\mathbf{x}, t)\right]}{\partial t}=0, \\
\left|q_{\tau}(\mathbf{x}, t)\right|=k_{\tau} q_{n}(\mathbf{x}, t) \Rightarrow \frac{\partial\left[u_{\tau}(\mathbf{x}, t)\right]}{\partial t}=-\frac{q_{\tau}(\mathbf{x}, t)}{\left|q_{\tau}(\mathbf{x}, t)\right|}\left|\frac{\partial\left[u_{\tau}(\mathbf{x}, t)\right]}{\partial t}\right| .
\end{gathered}
$$


The contact constraints (2)-(4) ensure that there is no interpenetration of the opposite crack faces, the normal component of the contact force is unilateral; and the opposite crack faces remain immovable with respect to each other in tangential direction while they are held by the friction force before the slipping occurs [16, 17, 20, 24].

Contact interaction makes the problem non-linear and the solution even for the case of harmonic loading becomes cyclic, but non-monoharmonic. That is why the normal and tangential components of the displacement discontinuity and the traction at the crack surface can be approximated by the following exponential Fourier series with respect to the time [16]:

$$
f(\bullet, t)=\operatorname{Re}\left\{\sum_{k=-\infty}^{+\infty} f^{k}(\bullet) e^{i \omega_{k} t}\right\}, \quad f^{k}(\bullet)=\frac{\omega}{2 \pi} \int_{0}^{T} f(\bullet, t) e^{-i \omega_{k} t} d t,
$$

where $\omega_{k}=2 \pi k / T$ and $i$ is the imaginary unit.

Thus, the system of boundary integral equations in the frequency domain can be represented as follows $\lceil 20,247$ :

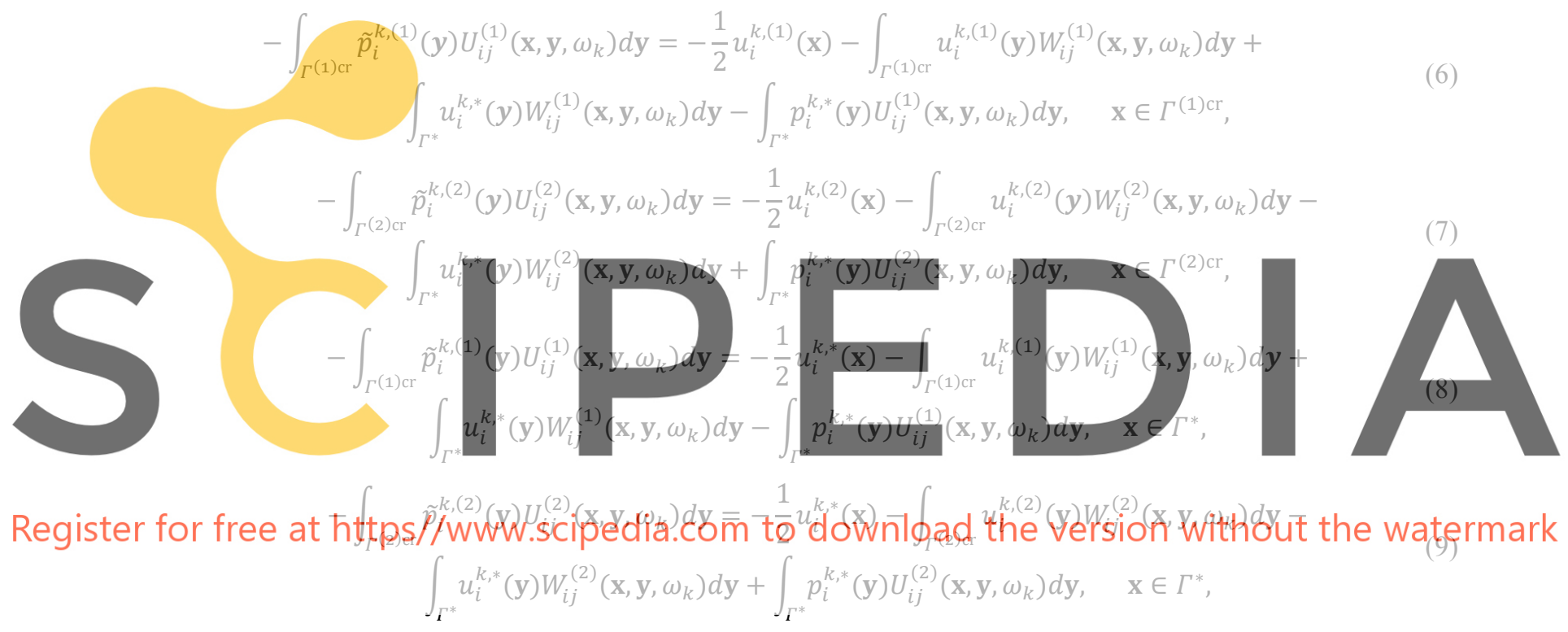

where $\tilde{p}_{i}^{k,(m)}(\mathbf{x}), p_{i}^{k, *}(\mathbf{x}), u_{i}^{k,(m)}(\mathbf{x})$ and $u_{i}^{k, *}(\mathbf{x})$ are the Fourier coefficients of the tractions and displacements at the crack's faces and the bonding interface.

Fundamental solutions in the frequency domain $U_{i j}^{(m)}\left(\mathbf{x}, \mathbf{y}, \omega_{k}\right)$ and $W_{i j}^{(m)}\left(\mathbf{x}, \mathbf{y}, \omega_{k}\right)$ have the following form $[1,9,12,16]$ :

$$
\begin{gathered}
U_{i j}^{(m)}\left(\mathbf{x}, \mathbf{y}, \omega_{k}\right)=\frac{1}{2 \pi \mu^{(m)}}\left(\psi^{(m)} \delta_{i j}-\chi^{(m)} \frac{\left(y_{i}-x_{i}\right)}{r} \frac{\left(y_{j}-x_{j}\right)}{r}\right), \\
W_{i j}^{(m)}\left(\mathbf{x}, \mathbf{y}, \omega_{k}\right)=\lambda^{(m)} n_{i}^{(m)}(\mathbf{y}) \frac{\partial}{\partial y_{k}} U_{k j}^{(m)}\left(\mathbf{x}, \mathbf{y}, \omega_{k}\right)+\mu^{(m)} n_{k}^{(m)}(\mathbf{y})\left[\frac{\partial}{\partial y_{k}} U_{i j}^{(m)}\left(\mathbf{x}, \mathbf{y}, \omega_{k}\right)+\right. \\
\left.\frac{\partial}{\partial y_{i}} U_{k j}^{(m)}\left(\mathbf{x}, \mathbf{y}, \omega_{k}\right)\right] .
\end{gathered}
$$

Here $\delta_{i j}$ is the Kronecker delta, $\lambda^{(m)}$ and $\mu^{(m)}$ are the Lamé coefficients, $r=\left|x_{1}-y_{1}\right|$ is the distance between the observation and load points. Functions $\psi^{(m)}$ and $\chi^{(m)}$ for the harmonic loading in $2 \mathrm{D}$ case are given as: 


$$
\psi^{(m)}=K_{0}\left(l_{2}^{(m)}\right)+\frac{1}{l_{2}^{(m)}}\left[K_{1}\left(l_{2}^{(m)}\right)-\frac{c_{2}^{(m)}}{c_{1}^{(m)}} K_{1}\left(l_{1}^{(m)}\right)\right], \quad \chi^{(m)}=K_{2}\left(l_{2}^{(m)}\right)-\left(\frac{c_{2}^{(m)}}{c_{1}^{(m)}}\right)^{2} K_{2}\left(l_{1}^{(m)}\right),
$$

where $l_{1}^{(m)}=i \omega_{k} r / c_{1}^{(m)}, l_{2}^{(m)}=i \omega_{k} r / c_{2}^{(m)} ; K_{n}(\bullet)$ is the modified Bessel function of the second kind and order $n$; and $c_{1}^{(m)}=\sqrt{\left(\lambda^{(m)}+2 \mu^{(m)}\right) / \rho^{(m)}}$ and $c_{2}^{(m)}=\sqrt{\mu^{(m)} / \rho^{(m)}}$ are the velocities of the longitudinal and transversal waves in the upper and lower half-spaces.

The appropriate system of linear algebraic equations (similar to the one presented in [7]) can be obtained from the system of boundary integral equations (6)-(9) and solved numerically using the iterative algorithm. At the first step the monoharmonic solution of the problem neglecting the effects of the crack closure and friction is obtained. Then the correction of the solution is performed applying the contact constraints (2)-(4) and the Fourier coefficients are gradually changed until the cyclic solution satisfying the constraints is found. The detailed analysis of the algorithm convergence for homogeneous and layered materials can be found in $[18,24]$. Note also that the appearing divergent integrals that depend on the type and order of the used space approximation shall be regularised and calculated. In the current study the simplest piecewise-constant approximation was used (as it successfully proved its efficiency for 2D problems comparing, for example, with the Galerkin method [19]).

The detailed investigation of the solution's convergence with respect to the number of Fourier coefficients used in series (5), the number of the boundary elements (and size of the elements in the vicinity of the crack's tips) and the number of time intervals has been presented
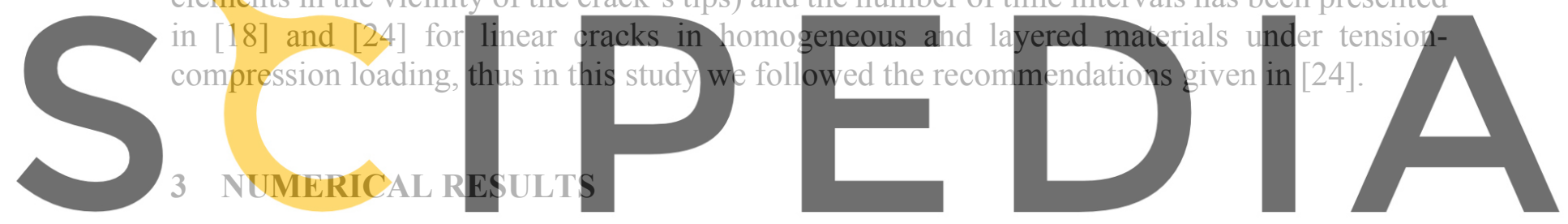

For the validation of the numerical model the crack of the length $2 L$ under the normal shear

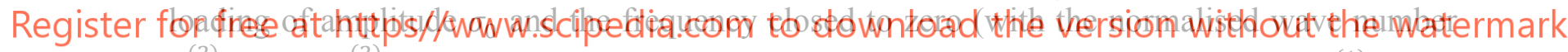

$\left.k_{2}^{(2)} L=\omega L / c_{2}^{(2)}=0.01\right)$ was considered. The mechanical properties of the material $\left(v^{(1)}=0.1\right.$, $E^{(1)}=29 \mathrm{GPa}$, and $\nu^{(2)}=0.49, E^{(2)}=400 \mathrm{GPa}$ ) were chosen to satisfy the following ratio [25]:

$$
\beta=\frac{\mu^{(2)}\left(\kappa^{(1)}-1\right)-\mu^{(1)}\left(\kappa^{(2)}-1\right)}{\mu^{(2)}\left(\kappa^{(1)}+1\right)+\mu^{(1)}\left(\kappa^{(2)}+1\right)}=0.5,
$$

where $E^{(m)}$ is the Young's modulus, $\kappa^{(m)}=3-4 v^{(m)}$, and $v^{(m)}$ is the Poisson's coefficient.

The distributions of the normal components of the displacements, $2 \mu_{0} u_{n} / \sigma_{0} L$, at the crack faces and the bonding interface at a half-period of oscillation are given in Figures 1-4 for different numbers of the correction steps in the absence of the friction $\left(k_{\tau}=0.0\right)$, note that [9]:

$$
\mu_{0}=\mu^{(1)} \frac{1-\gamma_{2}}{1+\kappa^{(1)}}, \quad \gamma_{2}=\frac{a_{1}}{2}-a_{2}, \quad a_{1}=\frac{\mu^{(1)}-\mu^{(2)}}{\mu^{(1)}+\kappa^{(1)} \mu^{(2)}}, \quad a_{2}=\frac{\kappa^{(1)} \mu^{(2)}-\kappa^{(2)} \mu^{(1)}}{2\left(\mu^{(2)}+\kappa^{(2)} \mu^{(1)}\right)} .
$$

Please note that after the correction (see Figure 4) the Signorini constraints (2) are satisfied on the entire surface of the crack, and the Sommerfeld conditions are satisfied at the infinity, so the iterative process effectively corrected the solution.

The normal components of the displacement jump and contact forces at the crack surface are presented in Figures 5 and 6 for different values of the friction coefficient. The contact forces 
and the size of the contact zone are compared with the model static solution by Comninou and Dundurs [25]. As one can see the results are in a very good agreement. It should be noted that the friction significantly affected the solution, changing the size of the contact zone and the distribution of the displacements and forces (even for the "quasi-static" case considered for the validation purposes).

Furthermore, increase in the frequency of the loading changes the solution even more (for comparison, please see [26]). As an example, the time distributions of the normal and tangential components of crack faces displacements are given in Figures $7-10$ for $k_{2}^{(2)} L=0.1, k_{\tau}=1.0$. Note the significant difference in the displacement amplitudes caused by the shear loading.

\section{CONCLUSIONS}

Computing and full parametric analysis of the normal opening and shear modes of the stress intensity factors for different friction coefficients, will be the next stage of this study. Please also note that the suggested boundary integral approach has been successfully applied for twodimensional impact contact problems for homogeneous and layered materials [27-29]. In the future it can be extended to three-dimensional fracture mechanics problems for cracked materials under arbitrary dynamic loading, and the special attention shall be paid to the coupling oscillation singularities in the vicinity of the interface crack front $[1-3,13,23]$.

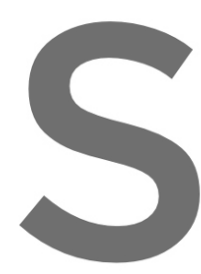

REFERENCES

Aliabadi, M.H., Mechanics. Comp Chai, H., Bao, Y. an
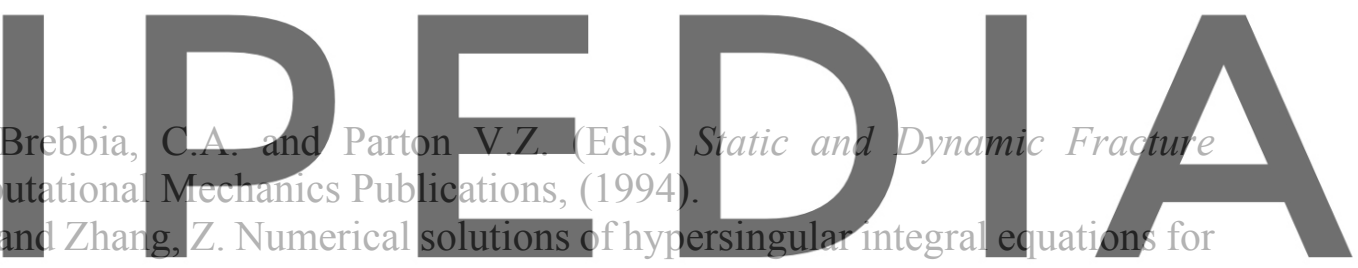
interface circular crack under axisymmetric loadings. Eng. Anal. Bound. Elem. (2021)

Register for free 35 t hittps//www.scipedia.com to download the version without the watermark

[3] Comninou M. An overview of interfacial cracks. Eng. Fract. Mech. (1990) 37(1):197-208.

[4] Golub, M. and Doroshenko, O. Boundary integral equation method for simulation scattering of elastic waves obliquely incident to a doubly periodic array of interface delaminations. $J$. Comput. Phys. (2019) 376:675-693.

[5] Guz, A.N., Guz, I.A., Men'shikov, A.V. and Men'shikov, V.A. Penny-shaped crack at the interface between elastic half-spaces under the action of a shear wave. Int. Appl. Mech. (2009) 45:534-539.

[6] Martin, P. Multiple Scattering. Interaction of Time-Harmonic Waves with N Obstacles. Cambridge University Press, (2006).

[7] Menshykov, O.V., Menshykov, V.A. and Guz, I.A. Elastodynamics of a crack on the bimaterial interface, Eng. Anal. Bound. Elem. (2009) 33:294-301.

[8] Men'shikov, V.A., Men'shikov, A.V. and Guz, I.A. Interfacial crack between elastic halfspaces under harmonic loading. Int. Appl. Mech. (2007) 43:865-873.

[9] Menshykova, M.V., Menshykov, O.V. and Guz, I.A. Linear interface crack under plane shear wave. CMES - Comput. Model. Eng. Sci. (2009) 48(2):107-120.

[10] Qu, J. Interface crack loaded by a time-harmonic plane wave. Int. J. Solids Struct. (1994) 31(3):329-345. 
[11] Mykhas'kiv, V.V., Zhbadynskyi, I.Ya and Zhang, Ch. On propagation of time-harmonic elastic waves through a double-periodic array of penny-shaped cracks. Eur. J Mech. A-Solid (2019) 73:306-317.

[12] Zhang, Ch. and Gross, D. On wave propagation in elastic solids with cracks. Computational Mechanics Publications, (1998).

[13] Comninou, M. and Schmueser, D. The interface crack in a combined tensioncompression and shear field. ASME. J. Appl. Mech. (1979) 46:345-348.

[14] Dhondt, G. Effect of contact between the crack faces on crack propagation. Key Eng. Mater. (2014) 577-578:61-64.

[15] Guz, A.N., Menshykov, O.V. and Zozulya, V.V. Surface contact of elliptical crack under normally incident tension-compression wave. Theor. Appl. Fract. Mech. (2003) 40:285-291.

[16] Guz, A.N. and Zozulya, V.V. Elastodynamic unilateral contact problems with friction for bodies with cracks. Int. Appl. Mech. (2002) 38:895-932.

[17] Guz, A.N. Nonclassical problems of fracture/failure mechanics: on the occasion of the 50th anniversary of the research (review). Int. Appl. Mech. (2019) 55:129-174.

[18] Menshykov, O.V., Menshykova, M.V. and Guz, I.A. Effect of friction of the crack faces for a linear crack under an oblique harmonic loading. Int. J. Eng. Sci. (2008) 46:438-458.

[19] Menshykov, O.V., Menshykova, M.V. and Wendland, W.L. On use of the Galerkin method to solve the fracture mechanics problem for a linear crack under normal loading. Int. Appl. Mech. (2005) 41:1324-1329.

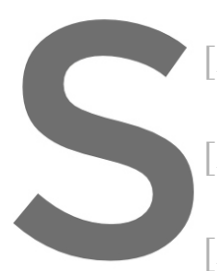

[20] Menshykova, M.V., Menshykov, O.V. and Guz, I.A.
interface crack under harmonic loading. In J. J. Fract. (201
[21] Menshykov, O.V., Men shykova, M.V. and Guz, I.A. 3-
for an interface crack under harmonic loading. Eng. Fract
[22] Mykhas'kiv, V. and Stankevych, V. Elastodynamic p with penny-shaped crack under harmonic torsion. Z. Angew. Math. Mech. (2019)

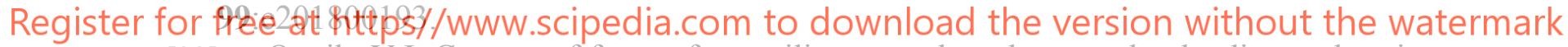

[23] Ostrik, V.I. Contact of faces of a rectilinear crack under complex loading and various contact conditions. Acta Mech. (2019) 230:3741-3758.

[24] Menshykova, M., Menshykov, O. and Guz, I.A. An iterative BEM for the dynamic analysis of interface crack contact problems. Eng. Anal. Bound. Elem. (2011) 35:735-749.

[25] Comninou, M. and Dundurs, J. Effect of friction on the interface crack loaded in shear. J. Elast. (1980) 10:203-212.

[26] Menshykov, O.V., Menshykov, V.A. and Guz, I.A. The effect of frequency in the problem of interface crack under harmonic loading. Int. J. Fract. (2007) 146:197.

[27] Menshykova, M.V., Menshykov, O.V., Guz, I.A., Wuensche, M. and Zhang, Ch. A boundary integral equation method in the frequency domain for cracks under transient loading. Acta Mech. (2016) 227:3305-3314.

[28] Menshykov, O.V., Menshykova, M.V. and Guz, I.A. Effects of crack closure and friction for linear crack under normal impact. Eng. Anal. Bound. Elem. (2020) 115:1-9.

[29] Menshykov, O.V., Menshykova, M.V. and Guz, I.A. Boundary integral equations in the frequency domain for interface linear cracks under impact loading. Acta Mech. (2020) 231:3461-3471. 


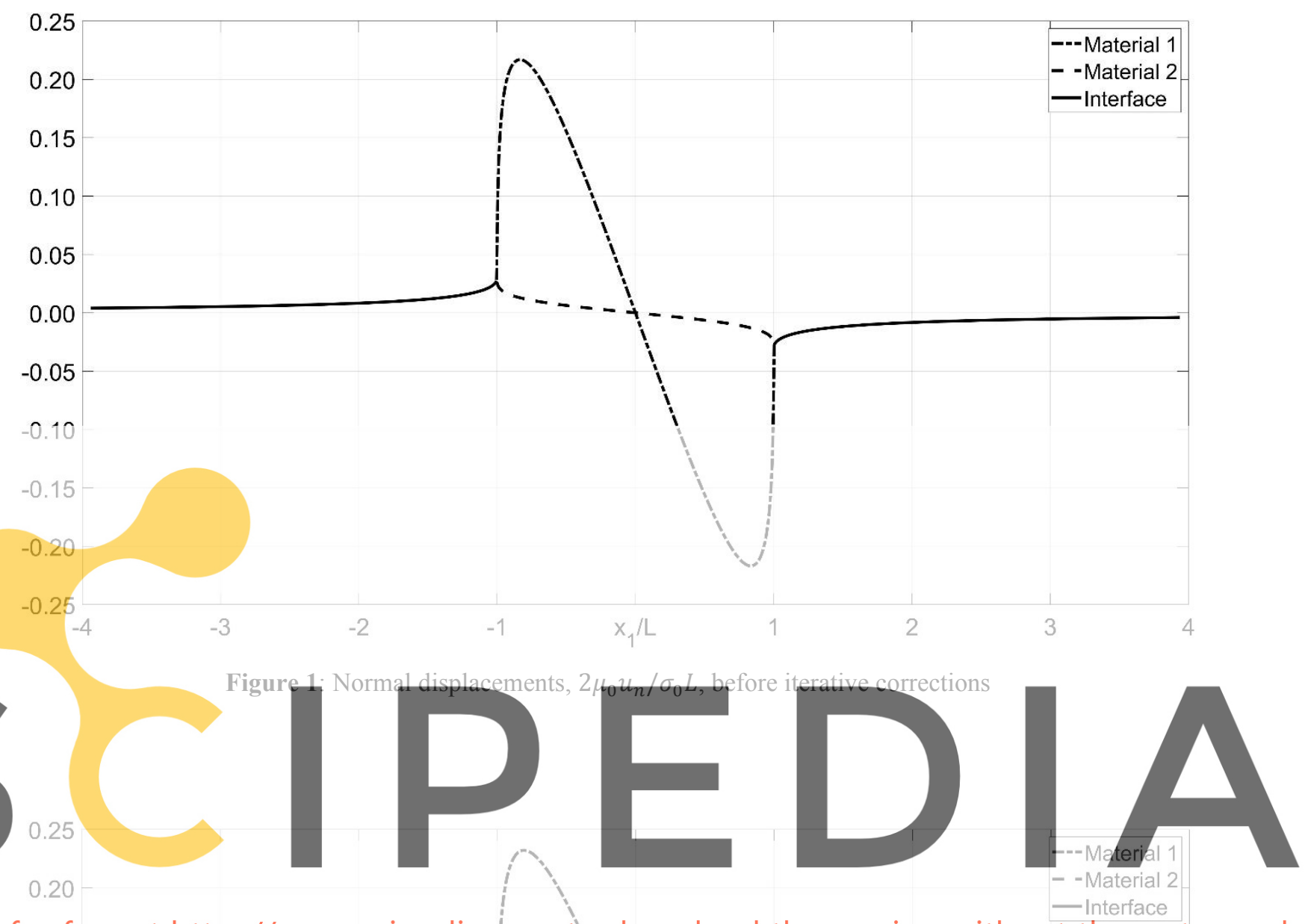

Register for free $_{1}$ at https//www.scipedia.łom to download the version without the watermark

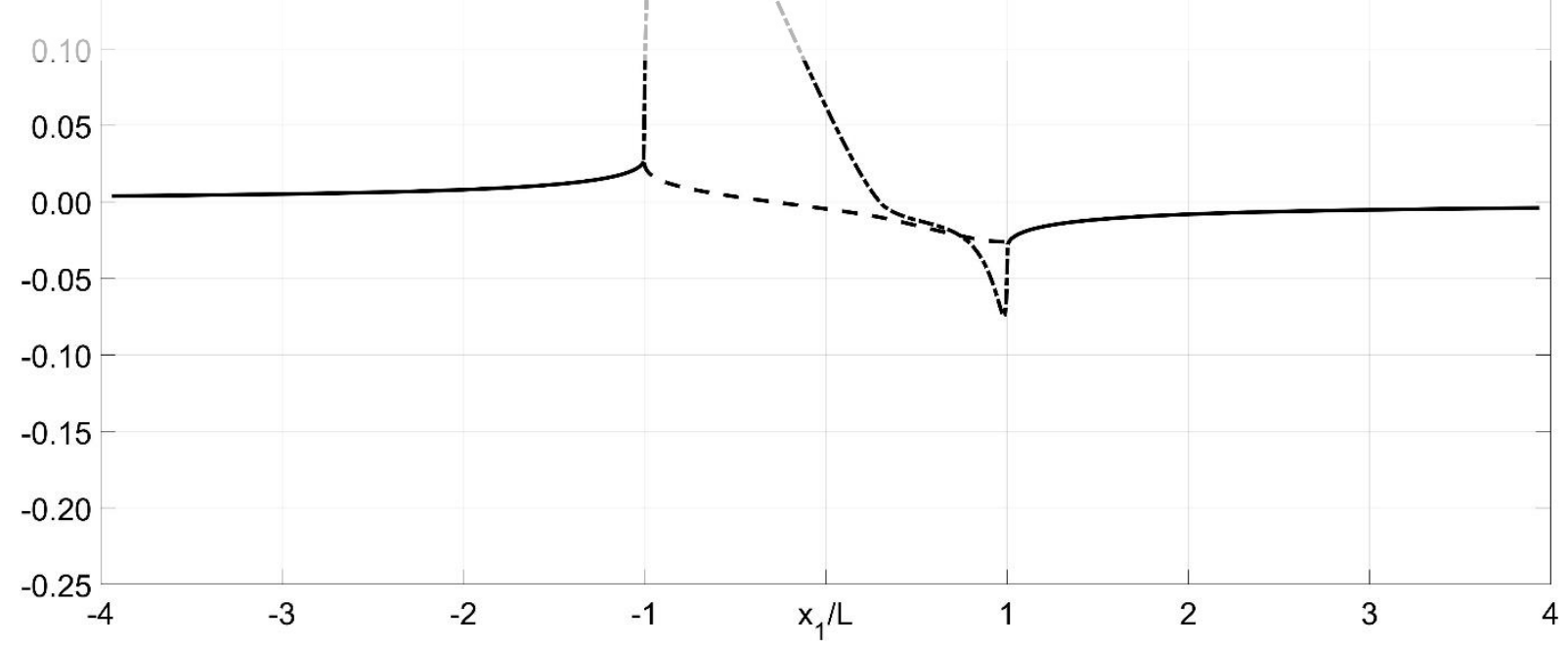

Figure 2: Normal displacements, $2 \mu_{0} u_{n} / \sigma_{0} L$, after 25 iterations 

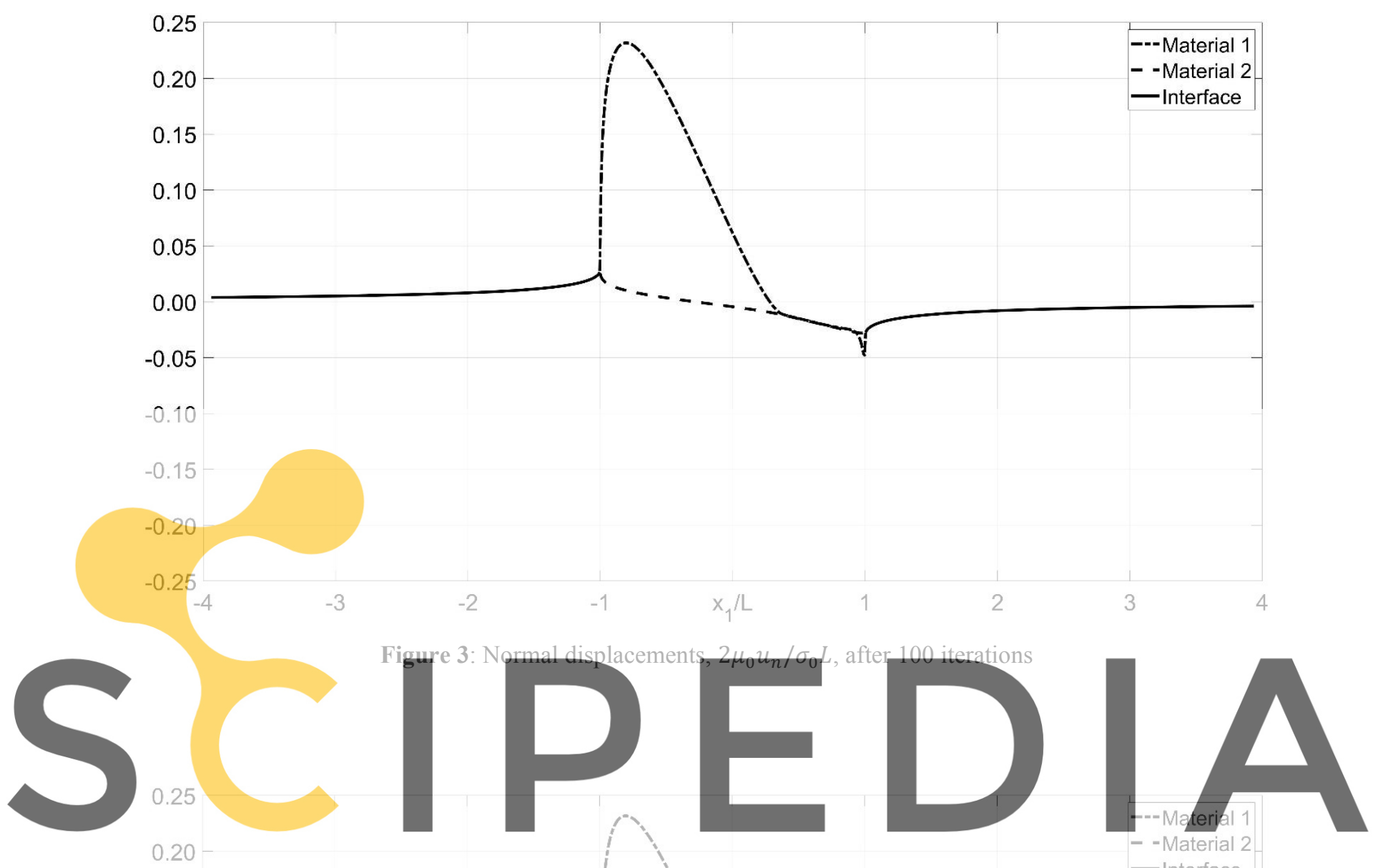

0.20

Register for $r_{1}$ ree at https//www.scipedia.łom to download the version without the watermark

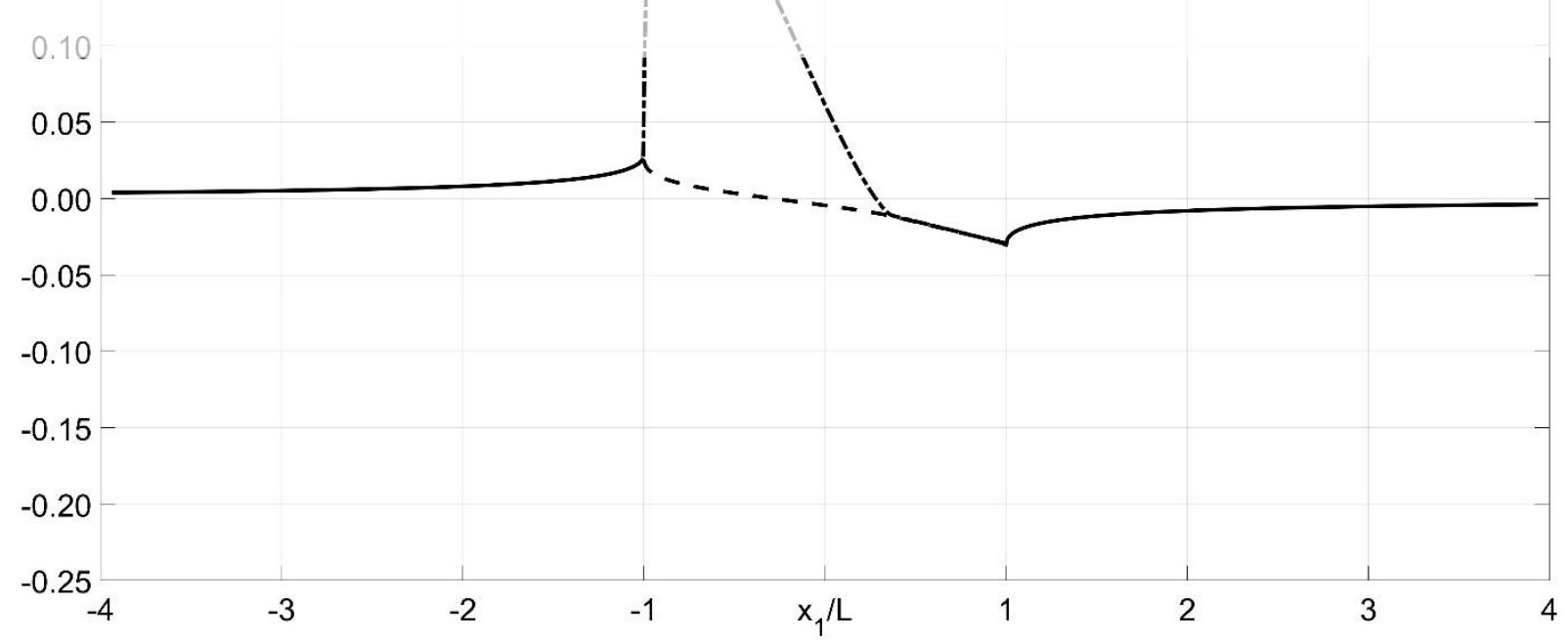

Figure 4: Normal displacements, $2 \mu_{0} u_{n} / \sigma_{0} L$, after 1000 iterations (correction is completed) 

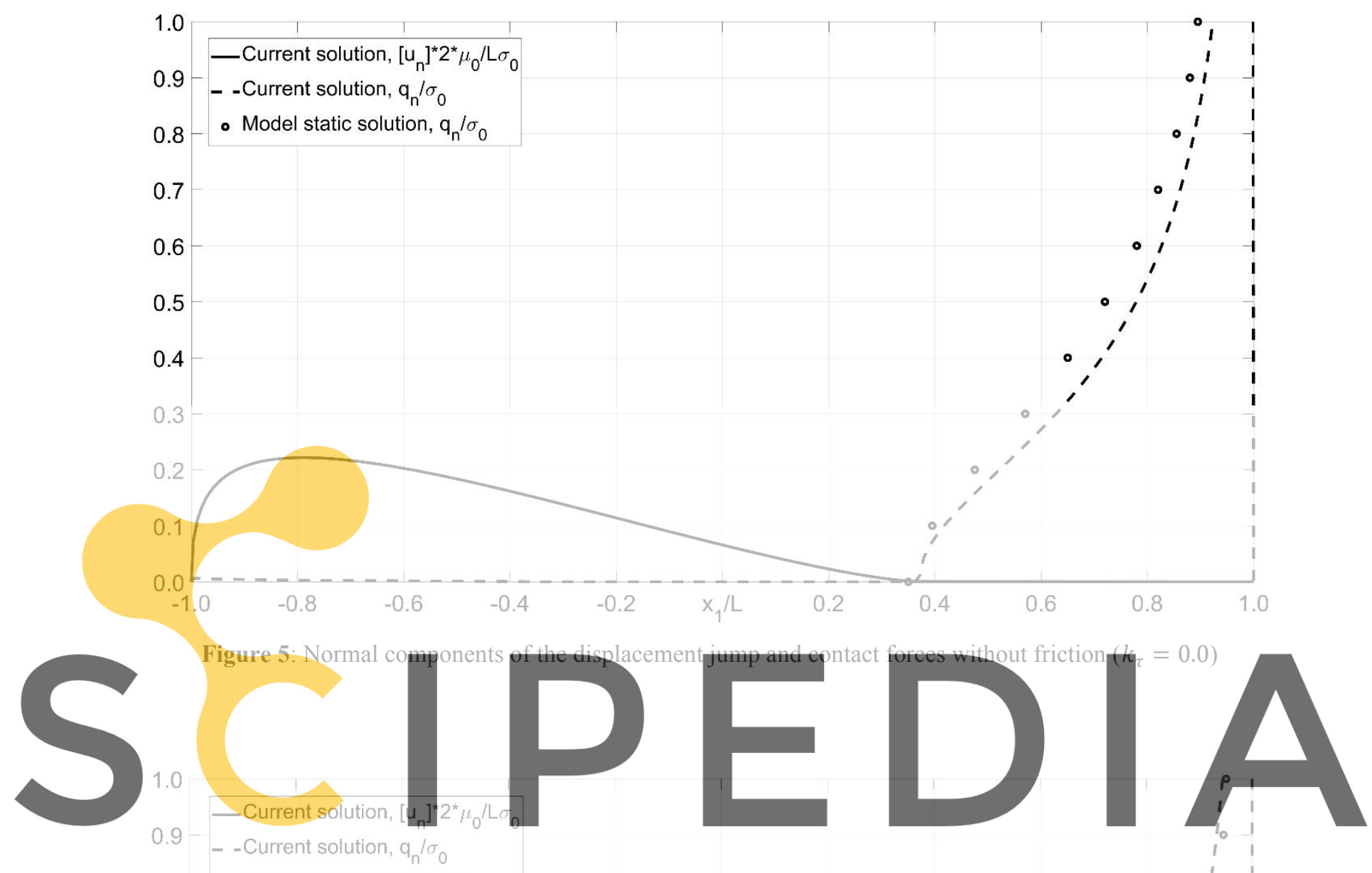

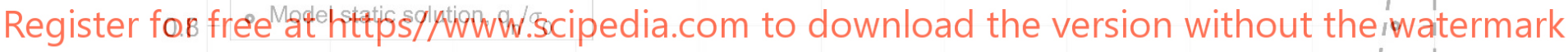

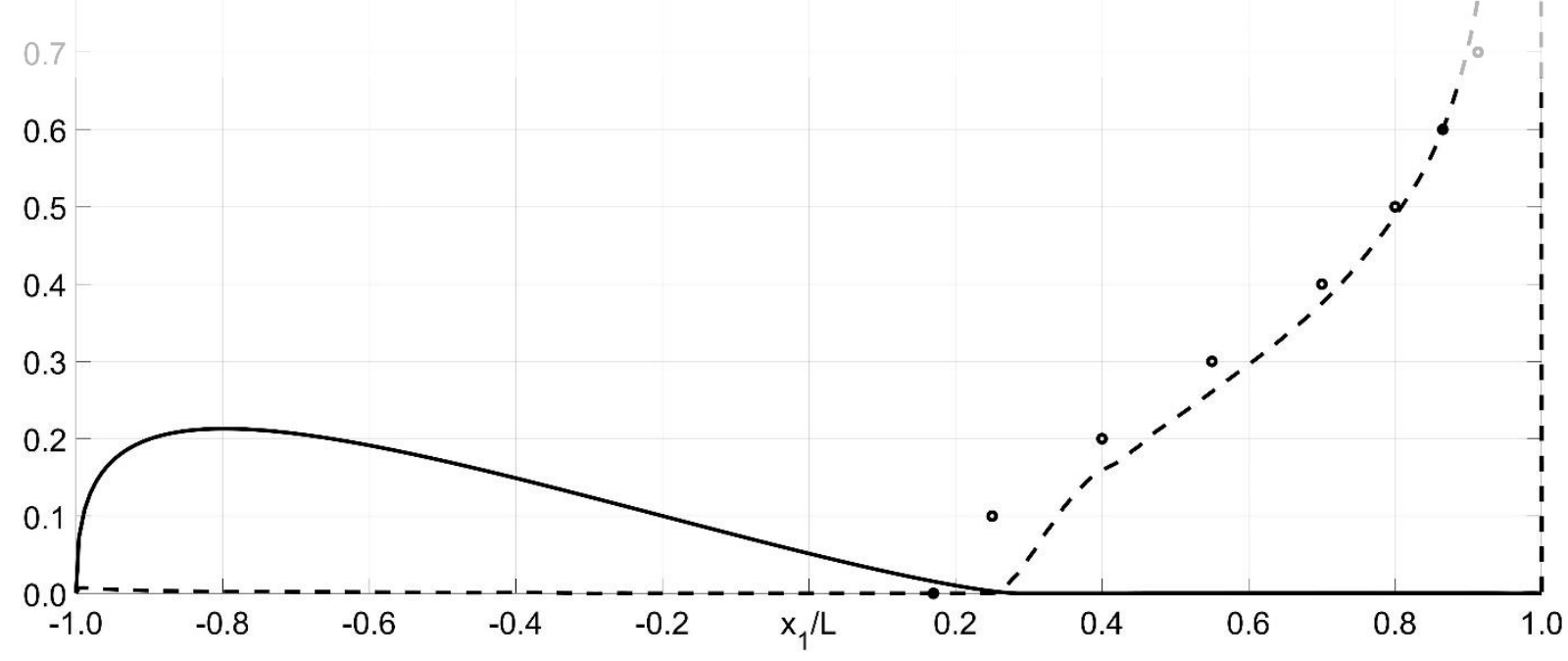

Figure 6: Normal components of the displacement jump and contact forces with friction $\left(k_{\tau}=1.0\right)$ 


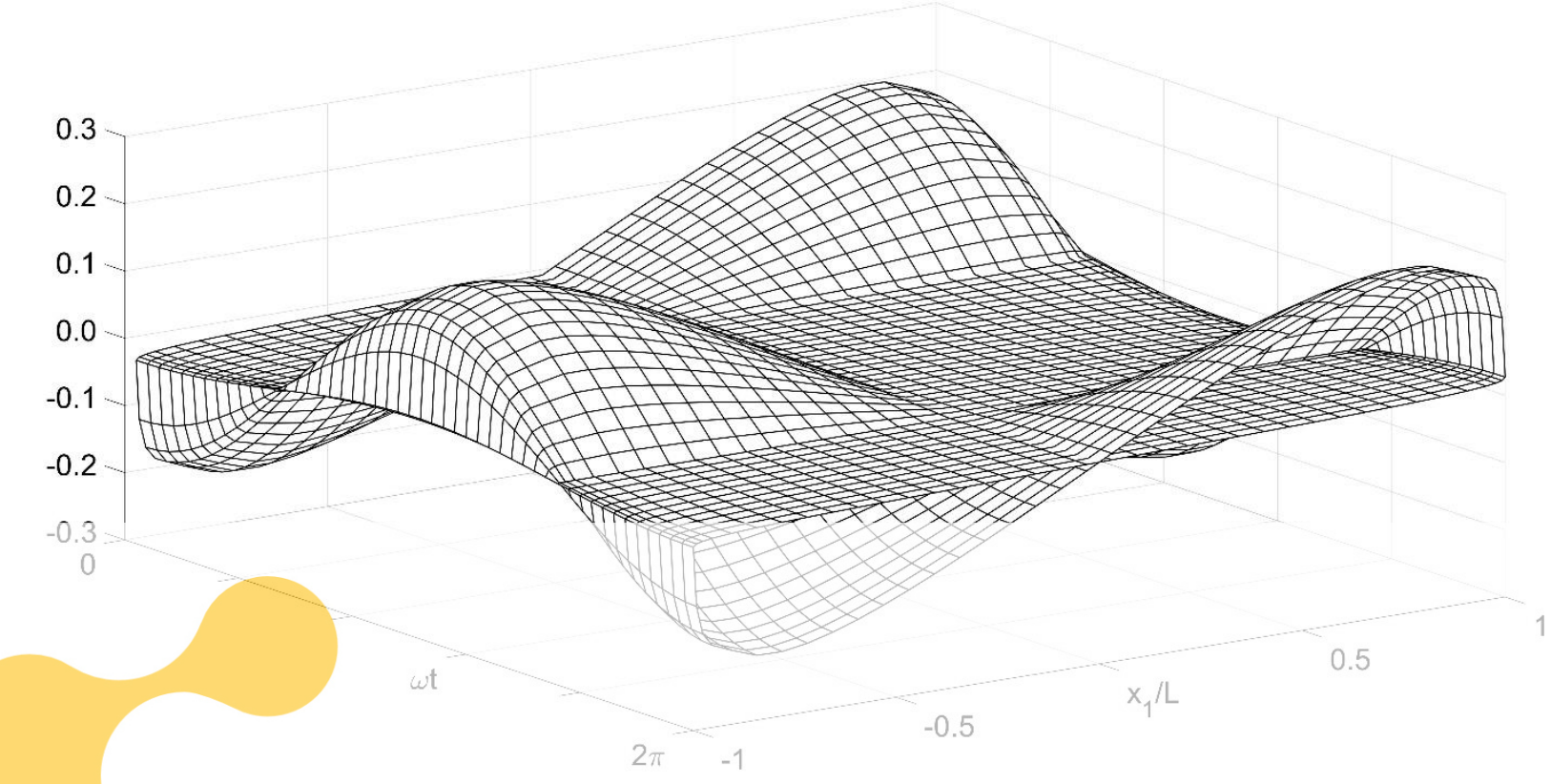

Figure 7: Normal components of the crack faces displacements before correction, $2 \mu_{0} u_{n} / \sigma_{0} L$
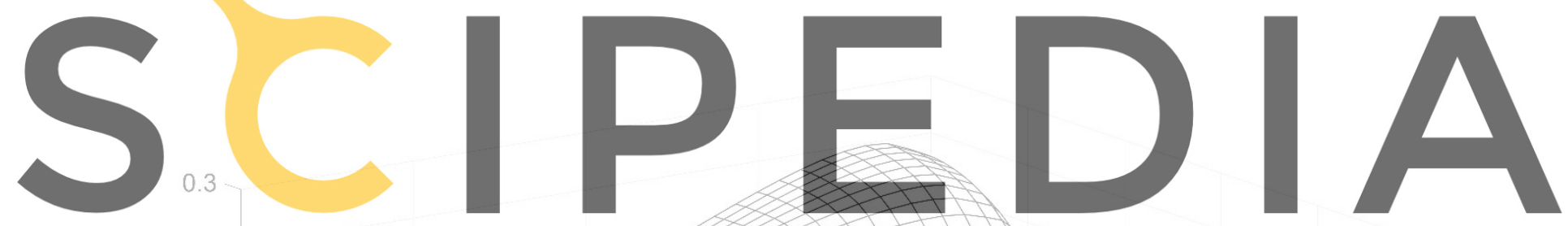

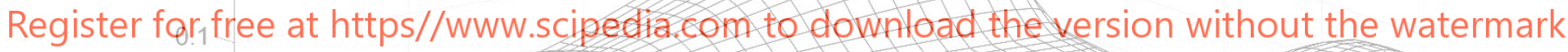

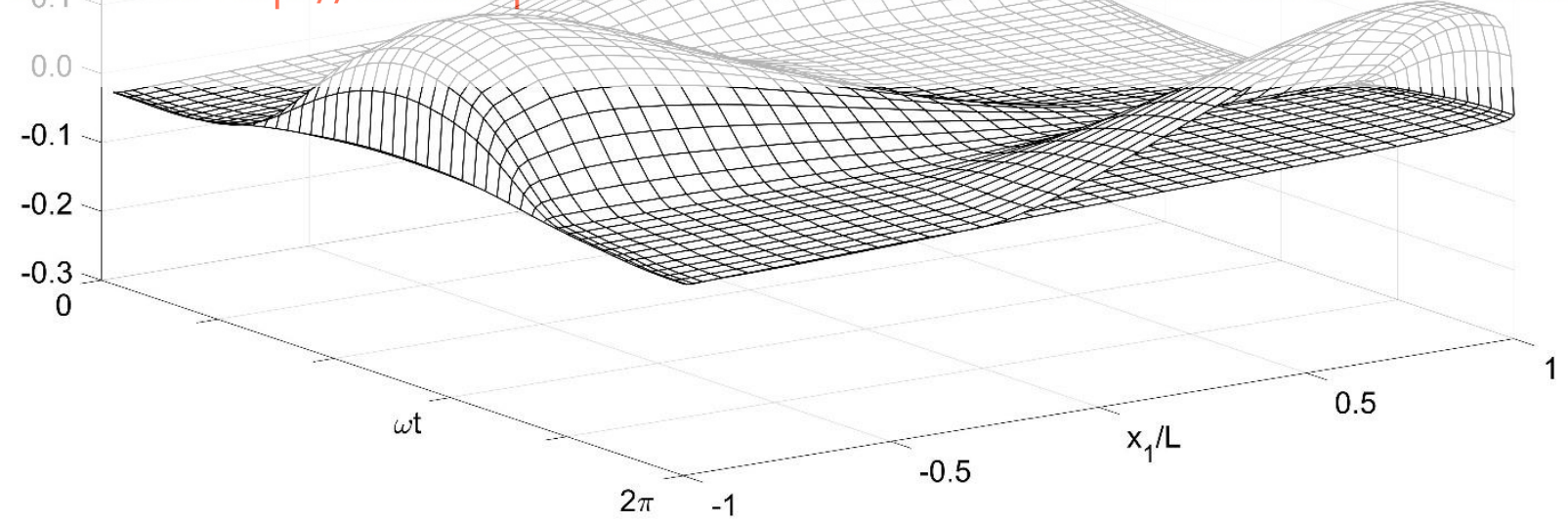

Figure 8: Normal components of the crack faces displacements after correction, $2 \mu_{0} u_{n} / \sigma_{0} L$ 


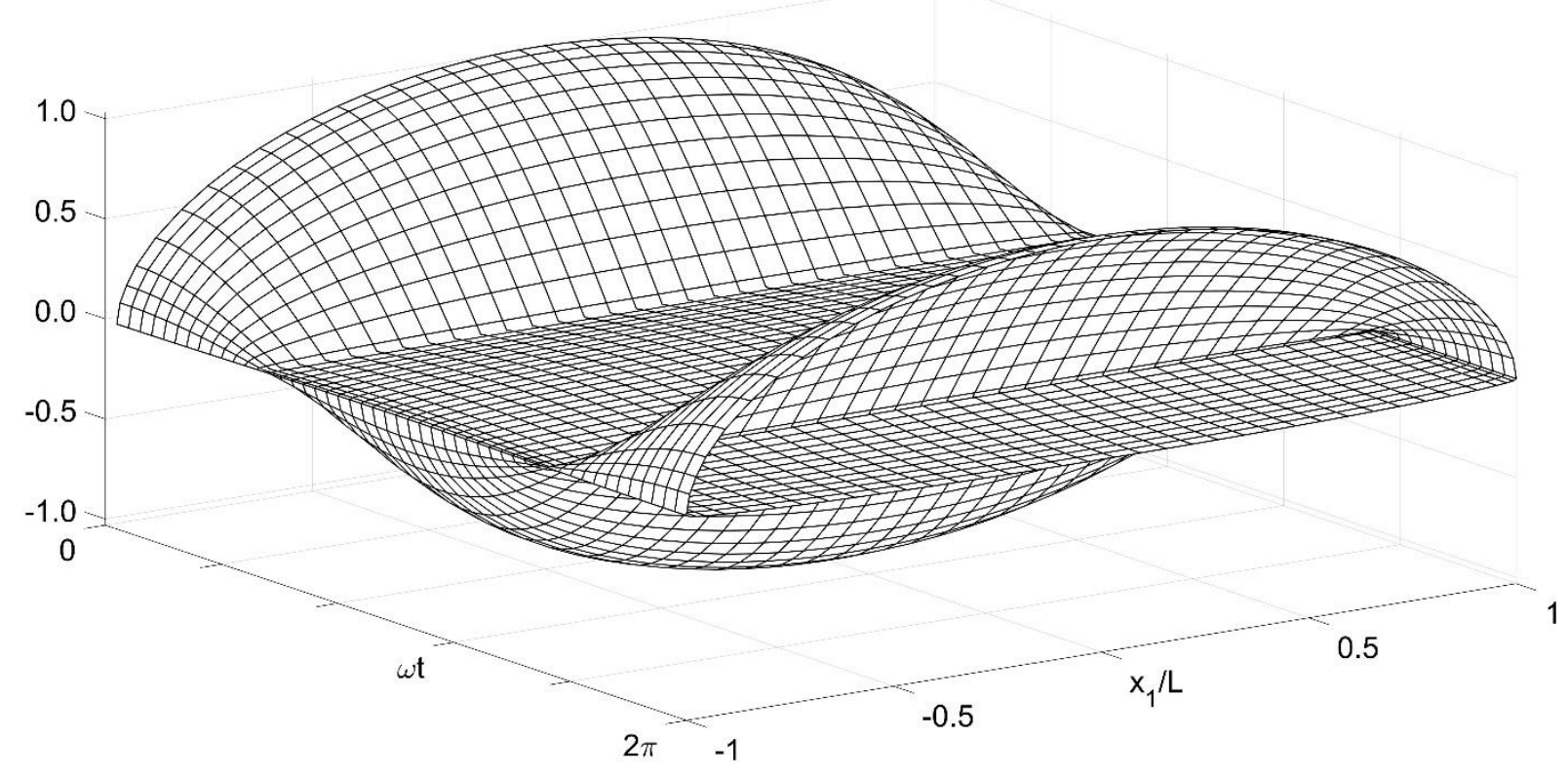

Figure 9: Tangential components of the crack faces displacements before correction, $2 \mu_{0} u_{\tau} / \sigma_{0} L$

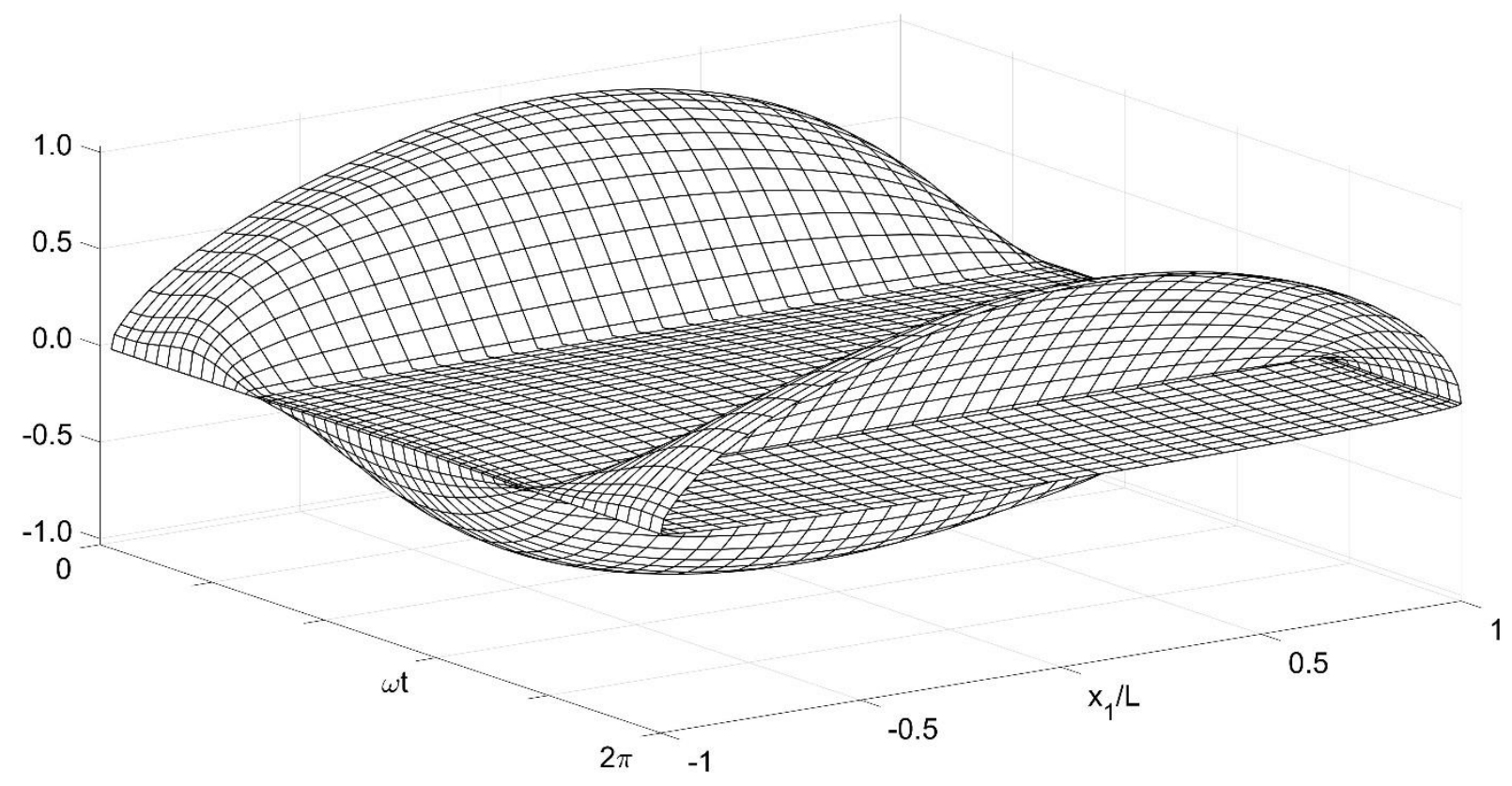

Figure 10: Tangential components of the crack faces displacements after correction, $2 \mu_{0} u_{\tau} / \sigma_{0} L$ 